\title{
Novel Surface Design of Deployable Reflector Antenna Based on Polar Scissor Structures
}

\author{
Pengyuan Zhao ${ }^{1,2,3}$, Jinguo $\mathrm{Liu}^{1,2^{*}}$, Chenchen Wu${ }^{1}$, Yangmin $\mathrm{Li}^{4}$ and Keli Chen ${ }^{1}$
}

\begin{abstract}
Space-deployable mechanisms can be used as supporting structures for large-diameter antennas in space engineering. This study proposes a novel method for constructing the surface design of space reflector antennas based on polar scissor units. The concurrency and deployability equations of the space scissor unit with definite surface constraints are derived using the rod and vector methods. Constraint equations of the spatial transformation for space n-edge polar scissor units are summarized. A new closed-loop deployable structure, called the polar scissor deployable antenna (PSDA), is designed by combining planar polar scissor units with spatial polar scissor units. The overconstrained problem is solved by releasing the curve constraint that locates at the end-point of the planar scissor mechanism. Kinematics simulation and error analysis are performed. The results show that the PSDA can effectively fit the paraboloid of revolution. Finally, deployment experiments verify the validity and feasibility of the proposed design method, which provides a new idea for the construction of large space-reflector antennas.
\end{abstract}

Keywords: Deployable structures, Polar scissor unit, Reflector antenna, Surface design

\section{Introduction}

Large-sized reflector antennas with high gain and narrow beam are widely used in space detection, radio astronomy, satellite communications, and other areas. Such an antenna often needs to be folded into a smaller volume before it is sent to space because of the size requirements of launch vehicles. After the spacecraft enters the orbit, the antenna is expanded to its working state. Therefore, using deployable structures to realize a parabolic profile is a key technology of the space reflector antenna [1-3]. The surface of the antenna is a wire mesh, and the geometrical control of the reflecting surface is achieved by adjusting the tension of the cable net. The HALCA (Highly Advanced Laboratory for Communications and Astronomy) scientific satellite, launched by the Japan Institute of Space Science and the National Astronomical

\footnotetext{
*Correspondence: liujinguo@sia.cn

${ }^{1}$ State Key Laboratory of Robotics, Shenyang Institute of Automation,

Chinese Academy of Science, Shenyang 110016, China

Full list of author information is available at the end of the article
}

Observatory of Japan, uses a radial extension antenna with many small triangular planes under tension to approximate the parabolic shape [4]. Furthermore, many scientists have studied the shape finding and pretightening force loading for cable membrane structures to achieve finer requirements for shape accuracy [5-8]. Although the scissor structure can also realize morphological adaptation in space, limited research has been conducted on its application in the field of large-scale space-deployable antennas.

The deployable scissor mesh enables fast and convenient wide-range transformation of shapes and volumes in response to environmental features with spatially variable requirements. Its basic components include two mutually intersecting rods, with the intersections connected by rotating joints [9]. The concept of the scissor structure was first introduced in 1961 by the Spanish architect Pinero. Then, Escrig and Valcarcel derived the developable conditions of the scissor structure, and compiled a matrix calculation program to analyze the motion and force of the structure $[10,11]$. Their study had a profound impact 
on subsequent studies [12, 13]. At present, the scissor unit is widely used in various fields of architecture, engineering and machinery with light weight and good mobility [14, 15]. The antenna of EGS (Experimental Geodetic Payload) sponsored by NASDA (The National Space Development Agency) is a scissor antenna consisting of a scissor-shaped circular support truss and a rib with tensioned film radiating from a central hub [16]. Bettini et al. [17] proposed a new lightweight structure based on the truss structure of a self-expanding scissor antenna with minimal mechanical connections. Gao et al. applied the scissor mechanism in robotics and designed a deployable manipulator for grasping objects with movement bifurcation [18]. Many structural parameters of the scissor mechanism were evaluated by Mira et al. [19]. Arnoutsa et al. [20] studied the geometric incompatibility in the development of bistable scissor structures. $\mathrm{Li}$ et al. [21, 22] conducted studies on the buckling analysis and deployment dynamics of a deployable structure based on the scissor unit. Akgüna et al. [23] proposed a structure with an adaptive scissor hinge that can be actuated between multiple curves using an actuator. Combining a scissor unit and a Hoekens linear link, Lu et al. [24] proposed a new deployable structure. Kwan [25] first introduced the scissor mechanism into the design of the parabolic deployable antenna and conducted a simple experiment. You and Pellegrino [26, 27] introduced a novel intermediate element in the middle of the scissor structure to provide greater freedom to the shape design of the deployable structure while maintaining its structural integrity. Combined with the cable net structure, this innovative structure can be used in the mesh reflector antenna. Roovers et al. [28, 29] pointed out that the scissor mechanism has considerable potential in profile design and proposed the aspherical geometric design method based on the generalized polar scissor unit. However, the scissor mechanism is applied mostly in-plane. Because of the limitation of geometric compatibility conditions, space structures based on the scissor unit are mainly restricted to the spherical structure or the specific geometry, which also significantly limits the application of the scissor mechanism in the space reflector antenna. There are a few studies that apply spatial polar scissor units in engineering, especially in the aerospace field.

In this study, polar scissor units is used in the space reflector antenna. A new method for the parametric design of the rotating surfaces is proposed by combining the space and the plane polar scissor units. The mathematical model of the spatial polar scissor unit is established in Section 2. The constraint equations of the scissor unit based on the rod length method are derived and vector method is proposed in Section 2. The two methods are compared, and the vector method is more suitable for solving the rod lengths of scissor units with definite surface constraints. In Section 3, a new method for the surface design of rotating paraboloids is proposed by using the derivations proposed in Section 2, which can realize a deployable structure that can form a target surface in the final state. The over-constrained problem is solved by releasing the curve constraint that locates at the end-point of the planar scissor mechanism. Kinematics and error analyses of the surface structure are performed. In Section 4, the lengths of rods are designed by the above method and the unfolding tests are carried out, which verifies validates the design method and provides new ideas for the application of the scissor mechanism in the space reflector antenna.

\section{Mathematical Model and Constraint Equations \\ 2.1 Types of Scissor Unit}

At present, there are three types of scissor units: translational scissor, polar scissor and angulated scissor units $[12,30]$. For the translational scissor unit, the unit lines that connect the upper and lower nodes must be parallel; they must remain in this state during deployment. For the polar scissor unit, the unit lines of the upper and lower nodes are converged at one point that is called the concurrency point. The angulated scissor unit was first proposed by Hoberman [31], and it is made up of two identical angled rods. The unit lines of this type also converge at the concurrency point. The three types of scissor units are shown in Figure 1.

The translational and polar scissor units must satisfy the application constraints given by Eq. (1), for them to be deployed and folded completely [13].

$$
a_{i}^{\prime}+b_{i}^{\prime}=a_{j}+b_{j} .
$$

\subsection{Spatial Polar Scissor Unit}

All the motion patterns of the three types of scissor units described in Section 2.1 are planar. If the hinge that connects the two scissor units is changed to a spherical hinge, the planar motion of the scissor unit can be changed into spatial motion. If the spatial polar scissor structure is required to be mobile, it is necessary to ensure that the extensions of the unit lines also converge

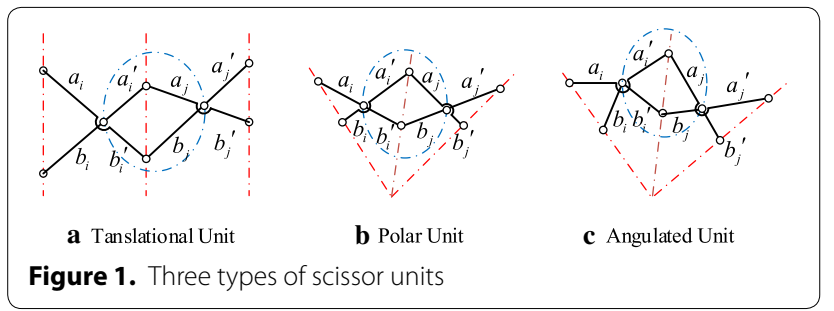




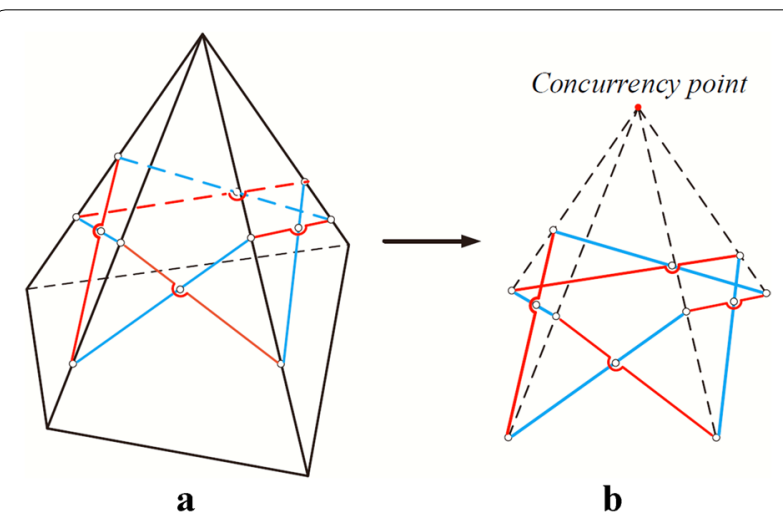

Figure 2. Pyramid structure of generalized spatial polar scissor unit $\mathbf{a}, \mathbf{b}$ is the construction process of spatial polar scissor units.

at the concurrency point. This is similar to placing the end of the scissor units on the ridgeline of a pyramid as shown in Figure 2, wherein, the pyramid apex is the concurrency point. The spatial polar scissor unit constructed by this method can realize spatial motion. If each group of adjacent scissor units satisfies (1), the scissor unit can be deployed and folded completely in space [29].

The spatial polar scissor unit significantly expands the range of application of the scissor mechanism. If the spatial polar scissor units are designed into a network with the concurrency points of all scissor units transmitted to the same point, the network of units can be deployed and folded synchronously. If an appropriate rod design is used for the spatial polar scissor units, it is possible to obtain a space surface structure that satisfies the requirements of deployability and foldability. The rods design method of the spatial polar scissor unit is derived theoretically in Sections 2.3.

\subsection{Constraint Equation of Spatial Polar Scissor Unit}

For the spatial polar scissor unit (1) achieve motion synchronization of the ends of each scissor unit and (2) be able to fold and deploy fully, a constraint relationship must be established. Two methods, namely the rod method (RM) and the vector method (VM), are introduced to establish the constraint equations of spatial polar scissor units. In VM, the rod lengths of all scissor units are considered variables, and the constraint relationships between the scissor elements are represented by a trigonometric function. In VM, the endpoint coordinates of the scissor units are used as variables in the Cartesian coordinate system to represent the constraint relationships among the scissor elements. The two methods are essentially the same. The rod length method is more intuitive, whereas the vector method is more suitable when the spatial scissor mechanism has a certain requirement with the analytical shape. The derivation of the constraint equation for the spatial polar scissor units under the two methods are given below hereafter.

\subsubsection{Rod Method}

The dimension design method of the spatial scissor unit using a triangular mesh is deduced here. The triangular mesh is the simplest mesh that can form a closed-loop link. In case of a triangular mesh, the number of bars is the least and the theoretical calculation is the most convenient. Furthermore, the method of determining the size of the scissor unit with a triangular mesh can be generalized to a polygon mesh. To facilitate the mathematical description of the spatial scissor unit and the visualization of the graph, the triangular pyramid composed of the triangle mesh, $\triangle A B C$, and the concurrency point $P$ are disassembled into the same plane as shown in Figure 3. Note that the non-spherical surface is target surface and surface equations are known. Next, the triangle mesh from point $C$ is decomposed and expanded into the same plane. Because $C^{\prime}$ and $C$ represent the same point, $P C^{\prime}=P C$. The method for determining the size of the scissor unit under a single triangular mesh is described in detail below.

Points $A, B$ and $C$ are set as the upper vertices of the triangular scissor unit, and the planar expansion of the space triangle scissor unit is as shown in Figure 4. The figure shows that there are twelve unknown parameters for the three sets of spatial polar scissor units: $a_{11}, a_{12}, a_{21}, a_{22}, a_{31}$, $a_{32}, b_{11}, b_{12}, b_{21}, b_{22}, b_{31}$, and $b_{32}$. Because the spatial positional relationship of points $A, B$, and $C$, and the concurrency point $P$ are determined in the state shown in Figure 4 , the line passing through the endpoints of triangle mesh $A B$, $B C$, and $C^{\prime} A$, scissor angle $\varphi$, and deployed angle of surface $\gamma$ are known. The line connecting the endpoint of the scissor unit to $P$ is called a concurrency line, and is denoted as $l$ (shown by the dotted line in Figure 4). Clearly, the values of $l$ are known. These parameters determine the deployed state of the triangular scissor unit.

Scissor angle $\varphi$ is represented by existing parameters based on the cosine law [29]:

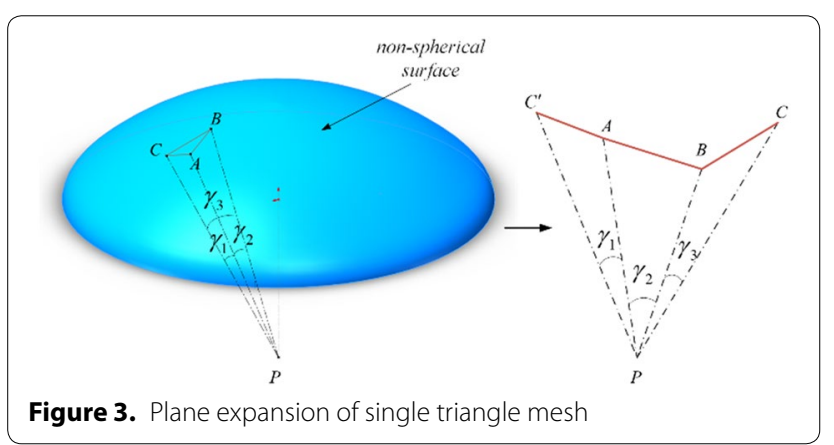




$$
\begin{aligned}
& C^{\prime} A^{2}=a_{11}^{2}+a_{12}^{2}-2 a_{11} a_{12} \cos \left(\pi-\varphi_{1}\right), \\
& \cos \varphi_{1}=\frac{l_{1}^{2}-a_{11}^{2}-a_{12}^{2}}{2 a_{11} a_{12}} .
\end{aligned}
$$

A derivation process similar to Eq. (2) can solve for $\cos \varphi_{2}$ and $\cos \varphi_{3}$. The deployability constraint needs to be satisfied to ensure that the spatial polar scissor unit can reach the minimum deployed state. Therefore, a set of deployability constraint equations is obtained:

$$
\left\{\begin{array}{l}
a_{12}+b_{12}=a_{21}+b_{21} \\
a_{22}+b_{22}=a_{31}+b_{31} \\
a_{32}+b_{32}=a_{11}+b_{11}
\end{array}\right.
$$

Three sets of closed-loop equations are established to ensure that the end positions of adjacent scissor units are the same:

$$
\left\{\begin{array}{l}
l_{1}=\left[\frac{\left(a_{12}+b_{11}\right) b_{12}}{a_{11} a_{12}-b_{11} b_{12}}+1\right] t_{1}=\left[\frac{\left(a_{32}+b_{31}\right) b_{32}}{a_{31} a_{32}-b_{31} b_{32}}+1\right] t_{4} \\
l_{2}=\left[\frac{\left(a_{11}+b_{12}\right) b_{11}}{a_{11} a_{12}-b_{11} b_{12}}+1\right] t_{2}=\left[\frac{\left(a_{22}+b_{21}\right) b_{22}}{a_{21} a_{22}-b_{21} b_{22}}+1\right] t_{2} \\
l_{3}=\left[\frac{\left(a_{21}+b_{22}\right) b_{21}}{a_{21} a_{22}-b_{21} b_{22}}+1\right] t_{3}=\left[\frac{\left(a_{32}+b_{31}\right) b_{32}}{a_{31} a_{32}-b_{31} b_{32}}+1\right] t_{3} .
\end{array}\right.
$$

The above process completes the derivation of the constraints of the spatial triangle scissor unit. If the rod length parameter satisfies Eqs. (3) and (7), the scissor unit can achieve mobility and complete deployment. When the scissor angles are $\varphi_{1}, \varphi_{2}$, and $\varphi_{3}$, as shown in Figure 4, the endpoints of the scissor unit are on the aspherical surface that we designed. If the spatial scissor unit only needs to satisfy only mobility and complete deployment, i.e., it has no deployed surface shape requirement, the constraints must be independent of $\varphi, \gamma$ and $l$. Therefore, Eq. (7) is simplified to:

$$
\left\{\begin{array}{l}
t_{1}^{2}=t_{4}^{2} \Rightarrow a_{11}^{2}+b_{11}^{2}-2 a_{11} b_{11} \cos \varphi_{1}=a_{32}^{2}+b_{32}^{2}-2 a_{32} b_{32} \cos \varphi_{3} \\
t_{2}^{2}=a_{12}^{2}+b_{12}^{2}-2 a_{12} b_{12} \cos \varphi_{1}=a_{21}^{2}+b_{21}^{2}-2 a_{21} b_{21} \cos \varphi_{2} \\
t_{3}^{2}=a_{22}^{2}+b_{22}^{2}-2 a_{22} b_{22} \cos \varphi_{2}=a_{31}^{2}+b_{31}^{2}-2 a_{31} b_{31} \cos \varphi_{3}
\end{array}\right.
$$

The deployed angles of the surface $\gamma_{1}, \gamma_{2}, \gamma_{3}$, are known parameters and can be expressed by the lengths of the rods and cosine of the scissor angles. Three sets of deployed angle formulas are obtained:

$$
\left\{\begin{array}{l}
\frac{\left(a_{12}+b_{11}\right) b_{12}}{a_{11} a_{12}-b_{11} b_{12}}=\frac{\left(a_{32}+b_{31}\right) b_{32}}{a_{31} a_{32}-b_{31} b_{32}} \\
\frac{\left(a_{11}+b_{12}\right) b_{11}}{a_{11} a_{12}-b_{11} b_{12}}=\frac{\left(a_{22}+b_{21}\right) b_{22}}{a_{21} a_{22}-b_{21} b_{22}} \\
\frac{\left(a_{21}+b_{22}\right) b_{21}}{a_{21} a_{22}-b_{21} b_{22}}=\frac{\left(a_{32}+b_{31}\right) b_{32}}{a_{31} a_{32}-b_{31} b_{32}}
\end{array}\right.
$$

$$
\left\{\begin{array}{l}
\cos \gamma_{1}=\cos \left(\delta_{12}-\rho_{11}\right)=\frac{a_{11} b_{12}+a_{12} b_{11}-\left(a_{11} a_{12}+b_{11} b_{12}\right) \cos \varphi_{1}}{t_{1} t_{2}} \\
\cos \gamma_{2}=\cos \left(\delta_{22}-\rho_{21}\right)=\frac{a_{21} b_{22}+a_{22} b_{21}-\left(a_{21} a_{22}+b_{21} b_{22}\right) \cos \varphi_{2}}{t_{2} t_{3}} \\
\cos \gamma_{3}=\cos \left(\delta_{32}-\rho_{31}\right)=\frac{a_{31} b_{32}+a_{32} b_{31}-\left(a_{31} a_{32}+b_{31} b_{32}\right) \cos \varphi_{3}}{t_{3} t_{4}} .
\end{array}\right.
$$

Note that the expressions for the deployed angles are not unique; each expression does not affect the final constraint relationship. Scissor unit endpoints must be on concurrency lines, and $l$ are known parameters. Three sets of concurrency equations are obtained based on the relationships between edges and angles of triangles:

$$
\left\{\begin{array}{l}
l_{1}=s_{1}+t_{1}=s_{4}+t_{4}=\left(a_{12}+b_{11}\right) \frac{\sin \rho_{12}}{\sin \gamma_{1}}+t_{1}=\left(a_{31}+b_{32}\right) \frac{\sin \rho_{31}}{\sin \gamma_{3}}+t_{4}, \\
l_{2}=s_{2}+t_{2}=\left(a_{11}+b_{12}\right) \frac{\sin \rho_{11}}{\sin \gamma_{1}}+t_{2}=\left(a_{22}+b_{21}\right) \frac{\sin \rho_{22}}{\sin \gamma_{2}}+t_{2}, \\
l_{3}=s_{3}+t_{3}=\left(a_{21}+b_{22}\right) \frac{\sin \rho_{21}}{\sin \gamma_{2}}+t_{3}=\left(a_{32}+b_{31}\right) \frac{\sin \rho_{32}}{\sin \gamma_{3}}+t_{3} .
\end{array}\right.
$$

The sinusoidal relationship in Eq. (6) is expressed by the unknown parameters of the rod length:
The spatial polar scissor unit can be made movable and fully deployable by satisfying only Eqs. (3) and (8) if there is no shape constraint for the scissor mechanism. The above derivation process is also applicable to the spatial polygon scissor unit. Now, the formula for concurrency and deployability of the spatial n-edge scissor unit will be obtained by the recursive method. 
Figure 5 shows the planar decomposition of the spatial $n$-edge scissor unit. The circled part of the red frame is the $n$ sets of scissor units that form the closed loop. The hinges are disassembled sequentially from junctions $x_{1}$ and $x_{2 n}, x_{2 n+1}$ and $x_{4 n}$. The black dotted line in the figure shows the original connection of the spatial closed loop by scissor units. The derivation of the concurrency and deployability equations for the spatial $n$-edge scissor unit are given below.

In Figure $5, n \geq 3$ represents the number of sides of the polygon and the number of scissor unit groups. The deployability constraint is satisfied between adjacent scissor units, and the resulting deployability constraint equations are

$$
\left\{\begin{array}{l}
x_{1}+x_{2 n+1}=x_{2 n}+x_{4 n}, \\
x_{2}+x_{2 n+2}=x_{3}+x_{2 n+3} \\
\vdots \\
x_{2(i-1)}+x_{2 n+2 i-2}=x_{2 i-1}+x_{2 n+2 i-1} \\
\vdots \\
x_{2 n-2}+x_{4 n-2}=x_{2 n-1}+x_{4 n-1}
\end{array}\right.
$$

where $n$ is the number of deployability constraint equations, and $i$ is the $i$ th set of scissor unit $(1<i<n, i \in Z)$. The motion synchronization of the adjacent scissor unit must be ensured, and the concurrency constraint equations are

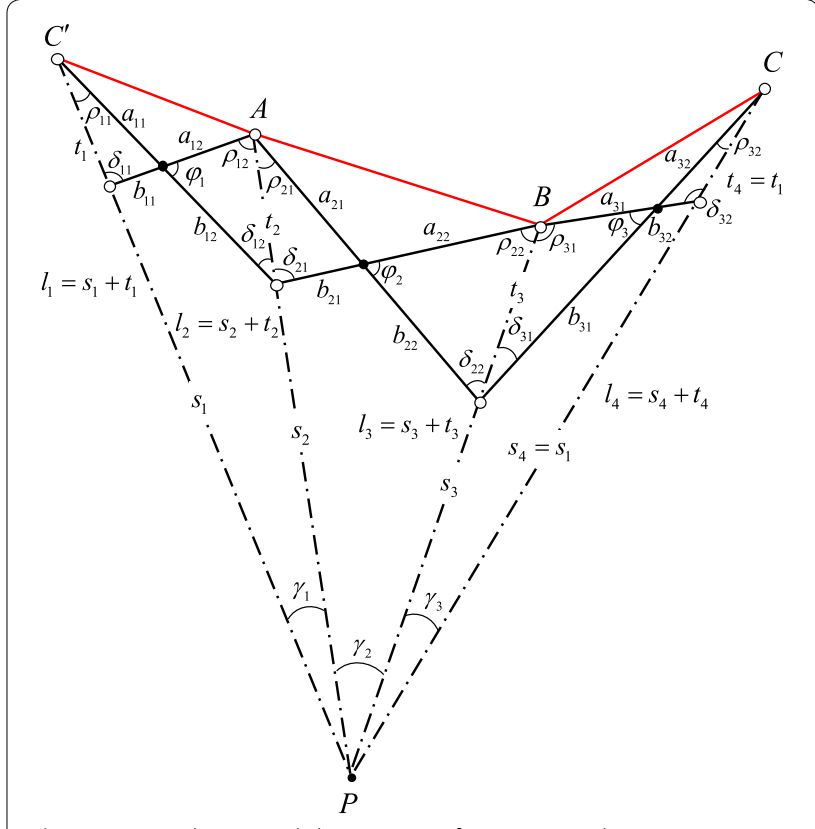

Figure 4. Mathematical description of space triangle scissor unit

constitutes spatial closed loop, and has superior versatility. The validity of the formula will be experimentally verified by designing the rod length in Section 4.1.

$$
\left\{\begin{array}{l}
\frac{\left(x_{1}+x_{2 n+2}\right) x_{2 n+1}}{x_{1} x_{2}-x_{2 n+1} x_{2 n+2}}=\frac{\left(x_{4}+x_{2 n+3}\right) x_{2 n+4}}{x_{3} x_{4}-x_{2 n+3} x_{2 n+4}} \\
\vdots \\
\frac{\left(x_{2 i-1}+x_{2(n+i)}\right) x_{2 n+2 i-1}}{x_{2 i-1} x_{2 i}-x_{2 n+2 i-1} x_{2(n+i)}}=\frac{\left(x_{2 i+2}+x_{2 n+2 i+1}\right) x_{2(n+i+1)}}{x_{2 i+1} x_{2 i+2}-x_{2 n+2 i+1} x_{2 n+2 i+2}} \\
\vdots \\
\frac{\left(x_{2 n-1}+x_{4 n}\right) x_{4 n-1}}{x_{2 n-1} x_{2 n}-x_{4 n-1} x_{4 n}}=\frac{\left(x_{2}+x_{2 n+1}\right) x_{2 n+2}}{x_{1} x_{2}-x_{2 n+1} x_{2 n+2}}
\end{array}\right.
$$

where $n$ is the number of concurrency constraint equations, and $i$ is the $i$ th set of scissor unit $(1<i<n, i \in Z)$. The first set of Eqs. (9) and (10) together form the closedloop equations of the spatial polar scissor unit

$$
\left\{\begin{array}{l}
x_{1}+x_{2 n+1}=x_{2 n}+x_{4 n}, \\
\frac{\left(x_{1}+x_{2 n+2}\right) x_{2 n+1}}{x_{1} x_{2}-x_{2 n+1} x_{2 n+2}}=\frac{\left(x_{4}+x_{2 n+3}\right) x_{2 n+4}}{x_{3} x_{4}-x_{2 n+3} x_{2 n+4}} .
\end{array}\right.
$$

Eq. (11) makes the scissor unit form a spatial closed loop and satisfies the constraints of concurrency and deployability. This general formula can be used to design the rod lengths of the polygon scissor unit that

\subsubsection{Vector Method}

Different from the derivations in Section 2.3.1, constraint equations for the spatial polar scissor unit in the Cartesian

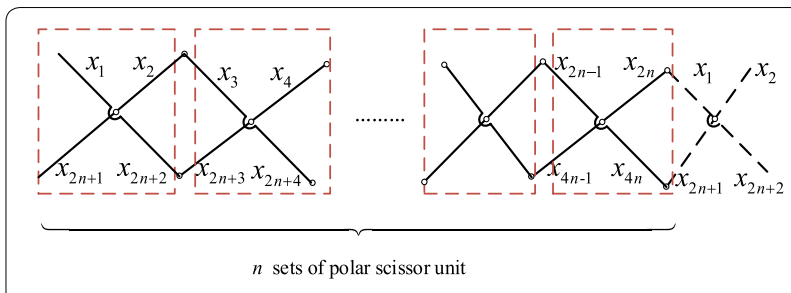

Figure 5. Planar decomposition of spatial $n$-edge polar scissor unit 
coordinate system are derived in this section. In the space coordinate system, the length of the rod cannot be directly represented and the endpoint coordinates of the scissor unit can be used as variables. The norm of the vector is used to represent the length of the rod after obtaining the coordinates of the endpoint. Considering the triangular scissor unit as an example, six coordinate points including eighteen parameters need to be expressed in the spatial coordinate system, as shown in Figure 6. The number of variables and the number of equations in the vector method are more than that in RM, and the solution process is more complex. However, the advantage of VM is that it can control the shape of the surface in the deployed state in a better manner.

The coordinates of the endpoints of the spatial triangle scissor unit are shown in Figure 6. In this state, the concurrency point is $P$, and concurrency constraint equations can be expressed as

$$
\left\{\begin{array} { l } 
{ \vec { A _ { 1 } P } / / \vec { A _ { 2 } P } } \\
{ \vec { B _ { 1 } P } P / / \vec { B _ { 2 } P } } \\
{ \vec { C _ { 1 } P } / / \vec { C _ { 2 } P } }
\end{array} \Rightarrow \left\{\begin{array}{l}
\frac{x_{a 1}-x_{p}}{x_{a 2}-x_{p}}=\frac{y_{a 1}-y_{p}}{y_{a 2}-y_{p}}=\frac{z_{a 1}-z_{p}}{z_{a 2}-z_{p}}, \\
\frac{x_{b 1}-x_{p}}{x_{b 2}-x_{p}}=\frac{y_{b 1}-y_{p}}{y_{b 2}-y_{p}}=\frac{z_{b 1}-z_{p}}{z_{b 2}-z_{p}}, \\
\frac{x_{c 1}-x_{p}}{x_{c 2}-x_{p}}=\frac{y_{c 1}-y_{p}}{y_{c 2}-y_{p}}=\frac{z_{c 1}-z_{p}}{z_{c 2}-z_{p}} .
\end{array}\right.\right.
$$

To represent the deployability constraint equations, we first need to express the coordinates of scissor points $D, E$, and $F$. Considering the coordinates of $D$ as an example, $D\left(x_{D}, y_{D}, z_{D}\right)$ is the intersection of vectors $\overrightarrow{A_{1} B_{2}}$ and $\overrightarrow{A_{2} B_{1}}$, therefore,

$$
\left\{\begin{array} { l } 
{ \vec { D A } / / / \vec { D B } _ { 2 } } \\
{ \vec { D A _ { 2 } / / D B _ { 1 } } }
\end{array} \Rightarrow \left\{\begin{array}{l}
\frac{x_{D}-x_{a 1}}{x_{D}-x_{b 2}}=\frac{y_{D}-y_{a 1}}{y_{D}-y_{b 2}}=\frac{z_{D}-z_{a 1}}{z_{D}-z_{b 2}}, \\
\frac{x_{D}-x_{a 2}}{x_{D}-x_{b 1}}=\frac{y_{D}-y_{a 2}}{y_{D}-y_{b 1}}=\frac{z_{D}-z_{a 2}}{z_{D}-z_{b 1}} .
\end{array}\right.\right.
$$

Solving the coordinates of the scissor point $D$ by Eq. (13):

$$
\left\{\begin{aligned}
x_{D} & =\frac{\left(x_{b 2} y_{a 1}-x_{a 1} y_{b 2}\right)\left(x_{b 1}-x_{a 2}\right)-\left(x_{b 1} y_{a 2}-x_{a 2} y_{b 1}\right)\left(x_{b 2}-x_{a 1}\right)}{\left(y_{a 1}-y_{b 2}\right)\left(x_{b 1}-x_{a 2}\right)-\left(y_{a 2}-y_{b 1}\right)\left(x_{b 2}-x_{a 1}\right)} \\
y_{D} & =\frac{\left(x_{b 2} y_{a 1}-x_{a 1} y_{b 2}\right)\left(y_{a 2}-y_{b 1}\right)-\left(x_{b 1} y_{a 2}-x_{a 2} y_{b 1}\right)\left(y_{a 1}-y_{b 2}\right)}{\left(x_{b 2}-x_{a 1}\right)\left(y_{a 2}-y_{b 1}\right)-\left(x_{b 1}-x_{a 2}\right)\left(y_{a 1}-y_{b 1}\right)} \\
z_{D} & =\frac{\left(x_{b 2} z_{a 1}-x_{a 1} z_{b 2}\right)\left(z_{a 2}-z_{b 1}\right)-\left(x_{b 1} z_{a 2}-x_{a 2} z_{b 1}\right)\left(z_{a 1}-z_{b 2}\right)}{\left(x_{b 2}-x_{a 1}\right)\left(z_{a 2}-z_{b 1}\right)-\left(x_{b 1}-x_{a 2}\right)\left(z_{a 1}-z_{b 1}\right)}
\end{aligned}\right.
$$

Similarly, the coordinates of the scissor points $E$ and $F$ can be obtained. The three sets of deployability constraint equations can be represented by the norms of the vector as

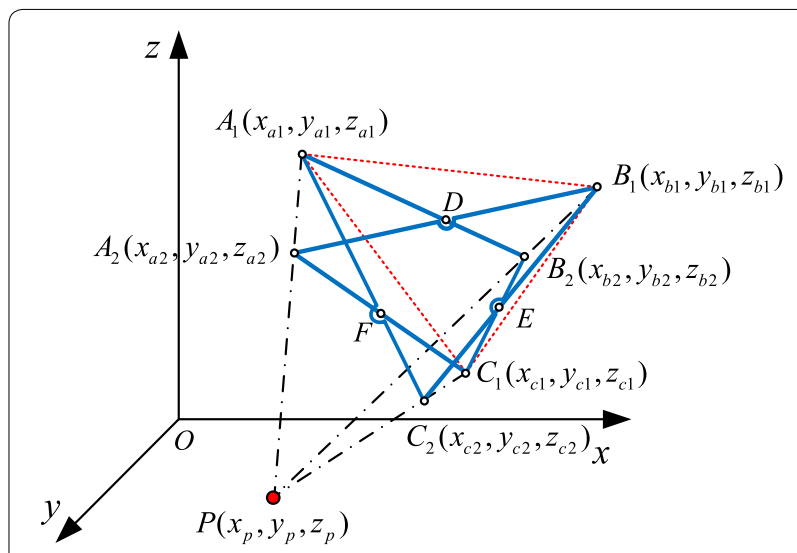

Figure 6. Mathematical description of vector method $\left(A_{1}-A_{3}, B_{1}-B_{3}\right.$, is the endpoints of the polar scissor unit and $D-F$ is scissor points)

$$
\left\{\begin{array}{l}
\left|\overrightarrow{D A_{1}}\right|+\left|\overrightarrow{D A_{2}}\right|=\left|\overrightarrow{F A_{1}}\right|+\left|\overrightarrow{F A_{2}}\right|, \\
\left|\overrightarrow{D B_{1}}\right|+\left|\overrightarrow{D B_{2}}\right|=\left|\overrightarrow{E B_{1}}\right|+\left|\overrightarrow{E B_{2}}\right|, \\
\left|\overrightarrow{E C_{1}}\right|+\left|\overrightarrow{E C_{2}}\right|=\left|\overrightarrow{F C_{1}}\right|+\left|\overrightarrow{F C_{2}}\right| .
\end{array}\right.
$$

If the spatial polar scissor unit satisfies Eqs. (12) and (15), mobility and complete deployment can be achieved in the Cartesian coordinate system. Because of the deployed state of the spatial scissor unit being independent of the position in the coordinate system, concurrency point $P$ can be moved to the origin of coordinates in practical applications to simplify the calculation.

\section{Surface Design Method}

The reflector system is perhaps the most widely used high-gain antenna, and its gain is considerably greater than $30 \mathrm{~dB}$ under normal conditions in the microwave band. The simplest reflector antenna consists of two parts: a reflective surface and a tiny fed antenna. The most popular form is a parabolic deployable reflector antenna (PDA) in space-oriented applications, which is a rotating paraboloid. Based on the scissor unit, the surface geometry design of the PDA is presented in this section. 


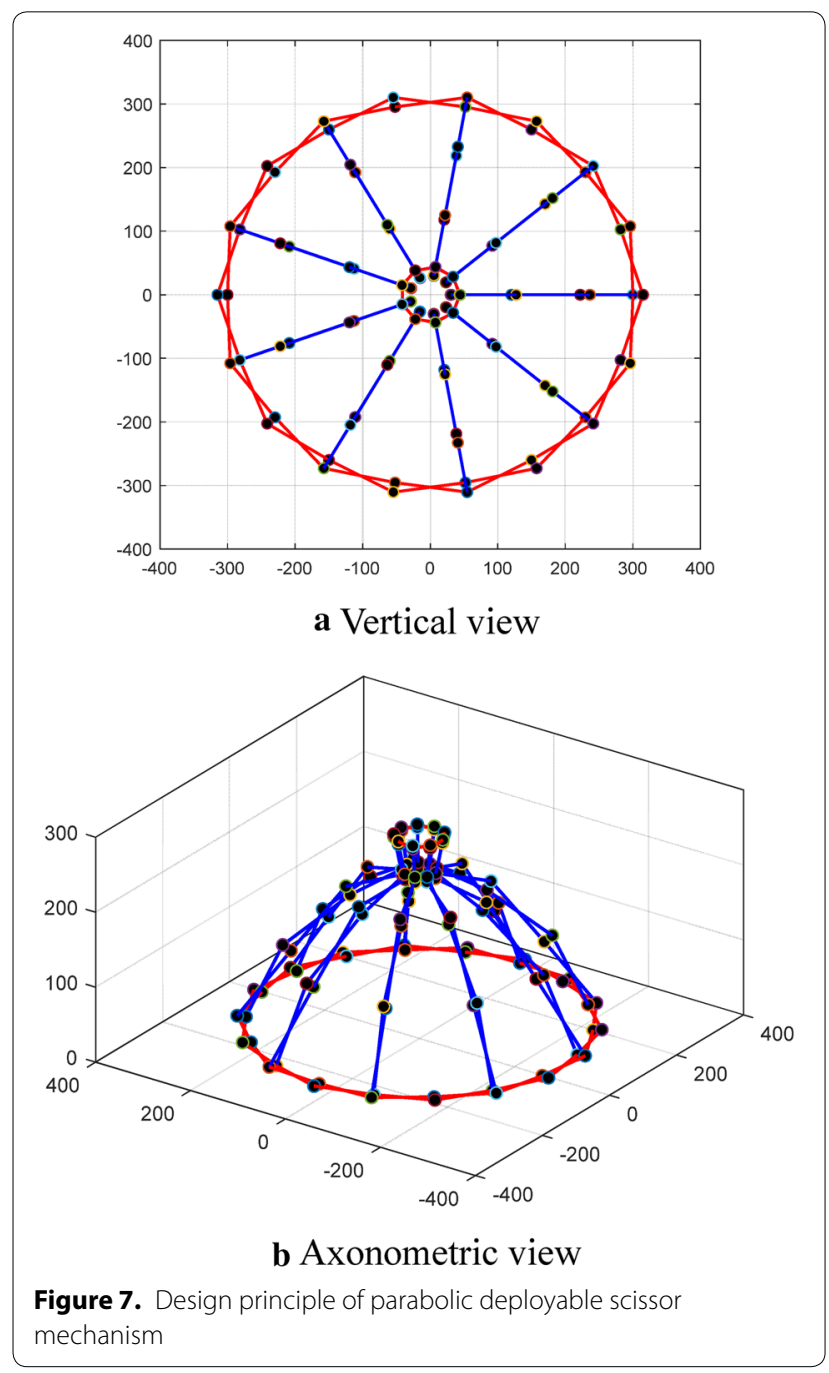

\subsection{Design Method}

PDA is a paraboloid of revolution formed by a parabola rotating along a central axis. It has a characteristic in that it is completely symmetrical, i.e., the all curves that the paraboloid is divided along any plane passing through the central axis to get the same parabola. Using this feature, we propose a new method for the parabolic design using a combination of planar and spatial polar scissor units. First, the planar polar scissor unit is used for the curve design so that the end-points of all scissor units on the same side coincide with the parabola in a certain state when the plane scissor mechanism is deployed. Second, the scissor units that are at both ends of the planar scissor mechanism are connected to two spatial polar scissor units (referred to as top and bottom rings), which together form a spatial closed-loop structure with a single degree of freedom as shown in Figure 7. The top ring, bottom ring and planar scissor mechanism are based on the polar scissor unit, and the two ends of the planar scissor mechanism are connected to the top and bottom rings. Therefore, the extension lines of the endpoint connection between all the scissor units intersect the same concurrency point to meet the condition of synchronous motion. If the mechanism meet the conditions of the deployability constraint on this basis, full deployment can be achieved.

\subsubsection{Bottom Ring (Top Ring) Design}

The structure of the rotating paraboloid consists of a planar scissor curve and the bottom and top rings that connect both its ends. As discussed in Section 2.2, there are concurrency and deployability constraints between the bottom ring, top ring, and planar scissor curve to ensure the validity of the designed structure. First, the dimensional design of the bottom ring is presented in this section. The design result will affect the size solution of the plane scissor curve, and the dimensions of the planar scissor curve will then be designed by the constraint relationship (see Section 3.1.2).

Depending on design requirements, the bottom and top rings will be designed as the spatial polar scissor unit mentioned in Section 2.2. More simply, in view of the complete symmetry of the rotating paraboloid, the bottom and top rings are regular polygonal structures, i.e., the structure of the rings can be determined by only a set of scissor units. Figure 7(a) shows the connection diagram of a set of planar scissor mechanisms and the bottom ring, where the blue color represents the planar scissor mechanism and the red color represents the bottom ring. For ease of description, the plane to which the planar scissor mechanism belongs is defined coincident with the $x o z$ plane. In this case, the $y$-coordinate of all the scissor units is 0 in the planar scissor mechanism. $S_{1}$ and $S_{6}$ are the two connection points of the planar scissor mechanism and bottom ring; $S_{6}$ is the point on the circular diameter of the rotating paraboloid. Because the aperture size of the antenna and the parabolic equation are known $(r=300 \mathrm{~mm}$ is chosen as the aperture value), the coordinates of $S_{6}$ are determined. To ensure that the concurrency constraint is satisfied, $S_{1}$ satisfies

$$
\frac{x_{1}}{z_{1}}=\frac{x_{6}}{z_{6}} \text {. }
$$

The rod length of the bottom ring is related to the number of equal divisions of the round circumference of the antenna. The higher number of divisions of the round aperture, the shorter the length of the rod. The top ring is similar to the bottom ring. However, considering the size limitation, if the top ring has too many aliquots, its rod length will be significantly short, which will affect the deployment process. In this paper, we divide the bottom 


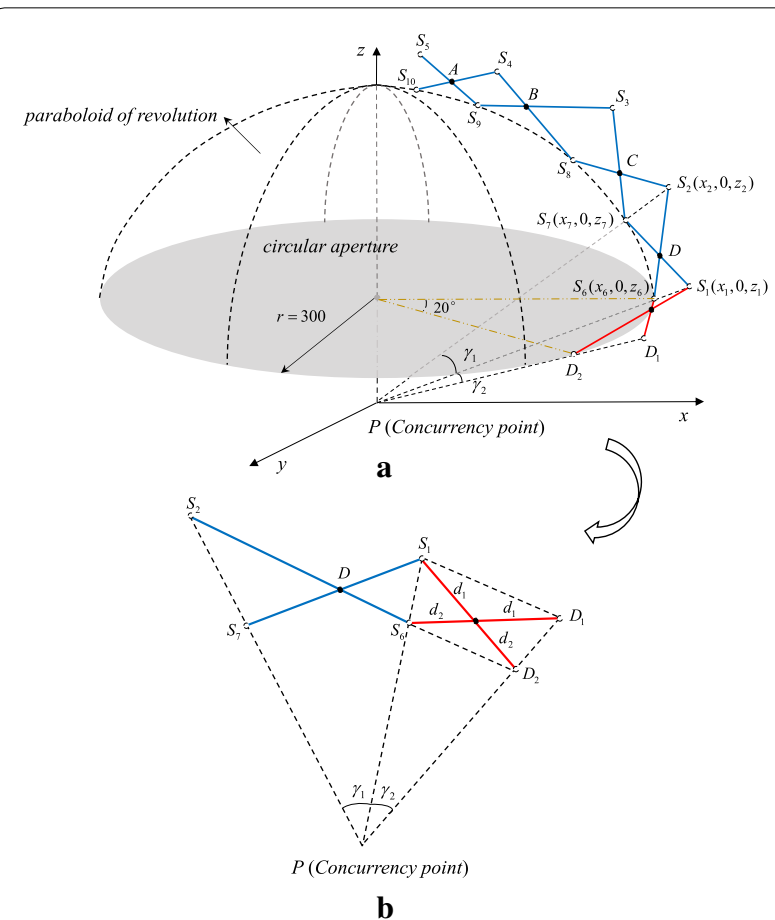

Figure 8. Connection between bottom ring and plane scissor mechanism: a connection of a planar scissor mechanism and a scissor unit of the bottom ring, $\mathbf{b}$ planar decomposition of the connection

ring into eighteen equal parts, and the top ring is divided into nine equal parts. That is, the bottom ring is made up of a spatial 18-edge polar scissor unit, and the top ring is made up of a spatial 9-edge polar scissor unit. Correspondingly, nine sets of planar scissor mechanisms are placed on the side of the PDA to form a rotating paraboloid, as shown in Figure 7(b). For the ease of description, the two sets of scissor units that connect the planar scissor mechanism and bottom ring are decomposed into the same plane, as shown in Figure 8. Because the scissor unit constituting the bottom ring is symmetrical, rods $D_{1} S_{6}$ and $D_{2} S_{1}$ are identical. Concurrency point $P$ is selected at the origin of coordinates. Then can be uniquely expressed as

$$
\cos \left(\gamma_{2}\right)=\frac{2 P S_{6}^{2}-D_{2} S_{6}^{2}}{2 P S_{6} \cdot D_{2} S_{6}}=\frac{\left(x_{6}^{2}+z_{6}^{2}\right)-2 r^{2} \sin ^{2}(\pi / 18)}{2 r \sqrt{x_{6}^{2}+z_{6}^{2}} \cdot \sin (\pi / 18)} .
$$

If the coordinate position of $S_{1}$ is determined, the rod length of the bottom ring can be uniquely determined by the similarity of triangles formula:

$$
\frac{D_{1} S_{1}}{D_{2} S_{6}}=\frac{P S_{1}}{P S_{6}}=\frac{d_{1}}{d_{2}} .
$$

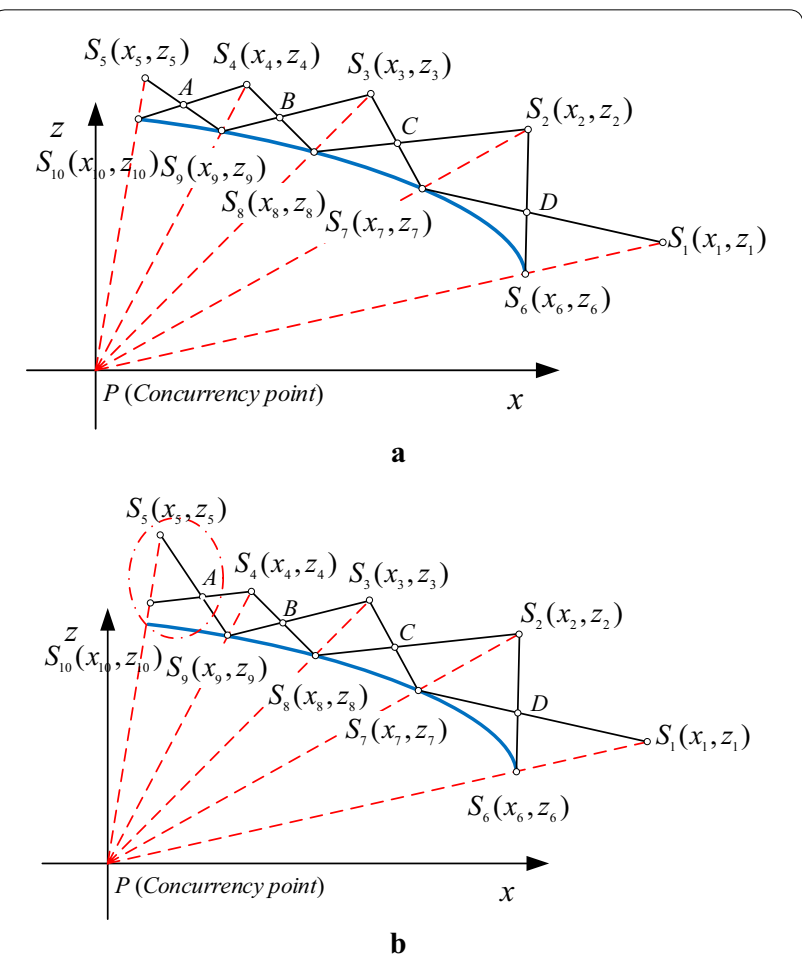

Figure 9. Planar scissor curve: a original planar scissor curve, b planar scissor curve that is released a curve constraint

Similarly, the size of the top ring can be obtained in the same way. After obtaining the size of the bottom ring, the deployability constraint needs to be satisfied to ensure complete deployment of the mechanism:

$$
D S_{1}+D S_{6}=d_{1}+d_{2} .
$$

In the same way, a similar deployability constraint is satisfied between the top ring and the planar scissor mechanism:

$$
A S_{5}+A S_{10}=l_{d},
$$

where $l_{\mathrm{d}}$ is the length of the scissor bar located on the top ring.

\subsubsection{Planar Scissor Mechanism}

The rod length design of the plane scissor mechanism of the PAD is described below. The parabolic equation is determined in the Cartesian coordinate system. To more easily fit the shape of the planar scissor mechanism in the specific deployed state, the VM is used to derive the constraint equation of the curve formed by the polar scissor unit. The planar scissor mechanism needs to satisfy the following three conditions in the specific deployed state: (1) concurrency and deployability constraints are met 
between all scissor units; (2) the end-points of all scissor units on the same side coincide with the parabola, which is called curve constraints, as $S_{6}-S_{10}$ in Figure 9(a); and (3) the two sets of scissor units at both ends of the planar scissor mechanism form a closed loop with the top and bottom rings, which needs to satisfy the deployability constraints of Eqs. (19) and (20).

The number of scissor units in top ring is assumed to be $n$ and the number of scissor units in planar scissor mechanism is assumed to be $m$. Therefore, the number of scissor units in bottom ring is $2 n$. We only study the number of constraints of a set of planar scissor mechanisms and its connections with the top and bottom rings. (1) Concurrency constraints $C_{c}$ : the planar scissor mechanism contains $m-1$ concurrency constraints and the connections with the top and bottom rings contains 2 concurrency constraints, so the number of $C_{c}$ is $m+1$. (2) Deployability constraints $C_{d}$ : the number of deployability constraints is same as the concurrency constraints, is $m+1$. (3) Curve constraints $C_{q}$ : because the lower endpoints of planar scissor mechanism coincide with the known parabola, the number of curve constraints is the same as the number of lower endpoints, which is $m+1$, so the number of constraints $C_{s}$ can be expressed:

$$
C_{s}=C_{c}+C_{d}+C_{q}=3 m+3 .
$$

If the planar scissor mechanism satisfies the above three conditions at the same time, there will be too many constraints and no solution. However, concurrency and deployability constraints must be satisfied. To solve this problem, we release endpoints of the planar scissor mechanism that connects with the top ring. That is, point $S_{10}$ does not coincide with the parabola, but the extension of the line connecting $S_{5}$ and $S_{10}$ intersects at $P$, as shown in Figure 9(b). For a large-diameter antenna, whether or not $S_{10}$ is coincident with the parabola has little effect if the rod lengths of scissor units connected to the top ring are sufficiently small and the distance from $S_{9}$ to the rotation axis of $z$ is sufficiently short. The derivation of the constraint equation for the planar scissor mechanism shown in Figure 9(b) given below.

$S_{6}-S_{9}$ are the points that coincide with the parabola and the appropriate coordinates can be determined according to design requirements. The remaining coordinate values are unknown. Take scissor unit $C$ and scissor unit $D$ as examples. Point $C$ is the intersection of vectors $\overline{S_{2} S_{8}}$ and vector $\overline{S_{1} S_{7}}$, so $\overline{C S_{2}} / / \overline{C S_{8}}$ and $\overline{C S_{3}} / / \overline{C S_{7}}$. Point $D$ is the intersection of vector $\overline{S_{2} S_{6}}$ and $\overline{S_{1} S_{7}}$, therefore, $\overline{D S_{1}} / / \overline{D S_{7}}$ and $\overline{D S_{2}} / / \overline{D S_{6}}$. The coordinates of points $C$ and $D$ can be obtained by the method presented in Section 2.3.2:

$$
\left\{\begin{aligned}
x_{C} & =\frac{\left(x_{2} z_{8}-x_{8} z_{2}\right)\left(x_{3}-x_{7}\right)-\left(x_{3} z_{7}-x_{7} z_{3}\right)\left(x_{2}-x_{8}\right)}{\left(z_{8}-z_{2}\right)\left(x_{3}-x_{7}\right)-\left(z_{7}-z_{3}\right)\left(x_{2}-x_{8}\right)}, \\
z_{C}= & \frac{\left(x_{2} z_{8}-x_{8} z_{2}\right)\left(z_{7}-z_{3}\right)-\left(x_{3} z_{7}-x_{7} z_{3}\right)\left(z_{8}-z_{2}\right)}{\left(x_{2}-x_{8}\right)\left(z_{7}-z_{3}\right)-\left(x_{3}-x_{7}\right)\left(z_{8}-z_{2}\right)} \\
x_{D} & =\frac{\left(x_{1} z_{7}-x_{7} z_{1}\right)\left(x_{6}-x_{2}\right)-\left(x_{2} z_{6}-x_{6} z_{2}\right)\left(x_{1}-x_{7}\right)}{\left(z_{7}-z_{1}\right)\left(x_{2}-x_{6}\right)-\left(z_{6}-z_{2}\right)\left(x_{1}-x_{7}\right)} \\
z_{D} & =\frac{\left(x_{1} z_{7}-x_{7} z_{1}\right)\left(z_{6}-z_{2}\right)-\left(x_{2} z_{6}-x_{6} z_{2}\right)\left(z_{7}-z_{1}\right)}{\left(x_{1}-x_{7}\right)\left(z_{6}-z_{2}\right)-\left(x_{2}-x_{6}\right)\left(z_{7}-z_{1}\right)} .
\end{aligned}\right.
$$

If scissor units $C$ and $D$ satisfy the deployability constraint, then

$$
\begin{aligned}
& \sqrt{\left(x_{C}-x_{2}\right)^{2}+\left(z_{C}-z_{2}\right)^{2}}+\sqrt{\left(x_{C}-x_{7}\right)^{2}+\left(z_{C}-z_{7}\right)^{2}} \\
& =\sqrt{\left(x_{D}-x_{2}\right)^{2}+\left(z_{D}-z_{2}\right)^{2}}+\sqrt{\left(x_{D}-x_{7}\right)^{2}+\left(z_{D}-z_{7}\right)^{2}} .
\end{aligned}
$$

If scissor units $C$ and $D$ satisfy the concurrency constraint, then

$$
\frac{x_{1}}{x_{6}}=\frac{z_{1}}{z_{6}}, \frac{x_{2}}{x_{7}}=\frac{z_{2}}{z_{7}}, \frac{x_{3}}{x_{8}}=\frac{z_{3}}{z_{8}} .
$$

There are also constraints between other sets of scissor elements as shown in Eqs. (23) and (24). Moreover, the two sets of scissor units at both ends of the planar scissor mechanism also need to satisfy the constraint Eqs. (19) and (20). The PDA composed of the planar scissor mechanism, top ring, and bottom ring can realize mobility and complete deployment under the above conditions.

\subsection{Motion and Error Analyses}

In this section, the motion path simulation and error analysis of PSDA will be carried out. First, a satellite often needs to carry loads to complete multiple on-orbit services in space. A kinematics analysis can effectively determine the working space of PSDA during the deployment to avoid interference with other loads on the satellite. Second, PSDA can ideally achieve the coincidence

Table 1. Rod length of planar scissor mechanism (mm)

\begin{tabular}{lcccccccc}
\hline Rod number & $\mathbf{1 - 7}$ & $\mathbf{2 - 6}$ & $\mathbf{2 - 8}$ & $\mathbf{3 - 7}$ & $\mathbf{3 - 9}$ & $\mathbf{4 - 8}$ & $\mathbf{4 - 1 0}$ & $\mathbf{5 - 9}$ \\
\hline Part 1 & 58.33 & 67.06 & 72.47 & 64.58 & 65.87 & 34.16 & 30.19 \\
Part 2 & 70.73 & 49.48 & 63.50 & 65.31 & 33.11 & 62.20 & 18.11 \\
Total length & 129.06 & 116.54 & 135.97 & 129.89 & 98.98 & 96.36 & 44.30 \\
\hline
\end{tabular}


of the lower ends of the planar scissor mechanisms with the paraboloid in a certain state, as shown in Figure 9(b). However, in practice, the machining accuracy and deformation caused by the induced stress during the deployment cause a shape error in the PSDA. Therefore, it is necessary to carry out an error analysis on the PSDA.

The smaller design aperture $(r=300 \mathrm{~mm})$ chosen in this study is for the ease of manufacturing and the parabolic expression to be satisfied after deployment is Eq. (25). It can be seen from the design process in Section 3.1 that the design method is applicable to any rotating paraboloid. If the verification experiment of this prototype can meet the requirements, this surface design method can be applied to larger aperture parabolic expressions. Considering the machining error of $0.01 \mathrm{~mm}$ as an example, we carried out the dimension design of the rods for PSDA. Because the lower end of the planar scissor mechanism contains all the points that are coincident with the paraboloid of rotation, it is not

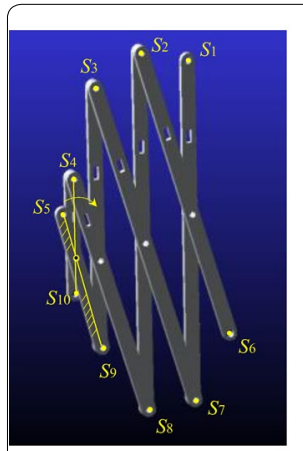

a Folded state drive is indicated in $\mathbf{a}$ )

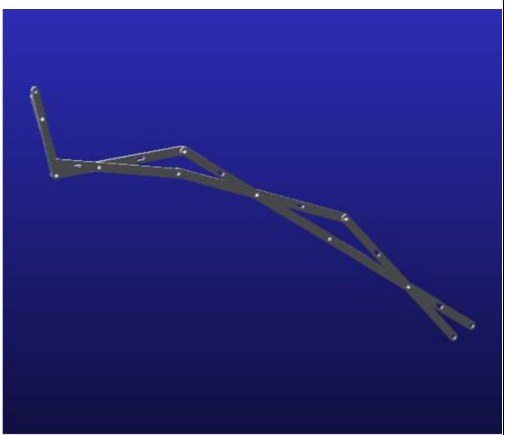

b Deployed state

d and necessary to target the overall structure for kinematic analysis and error analysis; instead one needs to target only for the planar scissor mechanism. In the deployed state selected in this paper, the ideal coordinate positions of $S_{6}, S_{7}, S_{8}$, and $S_{9}$ are $(300,20),(222,110.48),(120,188)$, $(30,218)$, respectively.

$$
z=-\frac{x^{2}+y^{2}}{450}+220 .
$$

Through MATLAB program calculation, the dimensional data of the planar scissor mechanism is presented in Table 1. The connection between $S_{1}$ and $S_{7}$ in Figure 9 (b) is recorded as rod 1-7 and the remaining part numbers are named in the same way. The portion of the upper-end of the scissor bar to the scissor point is designated Part 1, and the part of the lower-end point to the scissor point is designated Part 2.

When the trajectory fitting of the end of the scissor mechanism is performed, rod 5-9 is selected to be fixed, and the position of $S_{9}$ in the coordinate system is the same as that in which $S_{9}$ is located when the PSDA is deployed into a paraboloid. The hinge that connects rods 5-9 and 4-10 is set as driving, as shown in Figure 10(a). In practice, the PSDA cannot be completely deployed because of the thickness and shape of rods and the space occupied by hinges. Therefore, to fit the real situation, the angle between rods 5-9 and 4-10 does not start from $0^{\circ}$ during the simulation, rather it starts from a small angle. The initial scissor angle between 5-9 and 4-10 is set as $10^{\circ}$ in this paper, as shown in Figure 10(a). It is calculated that rod $4-10$ is rotated by $166.62^{\circ}$ in the clockwise direction and the planar scissor mechanism is deployed to the target state. Figure 10 shows the deployment process.
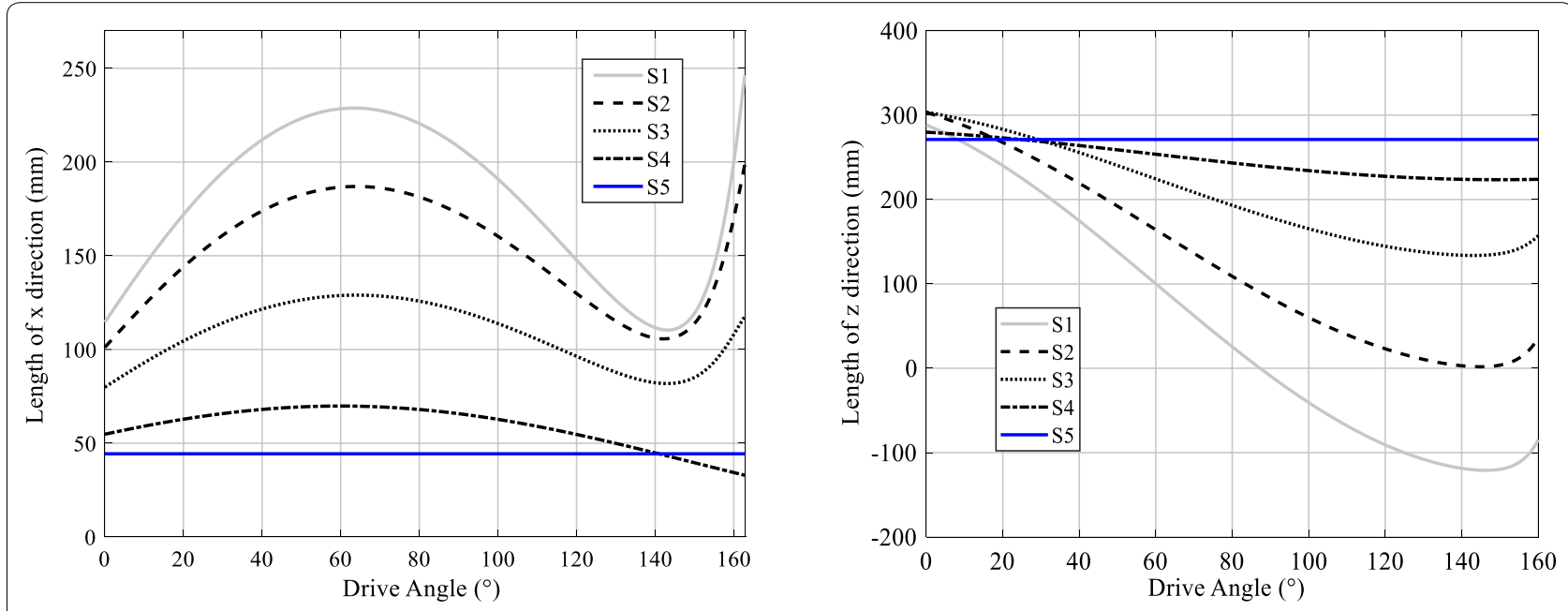

Figure 11. Motion trajectories of upper endpoints 

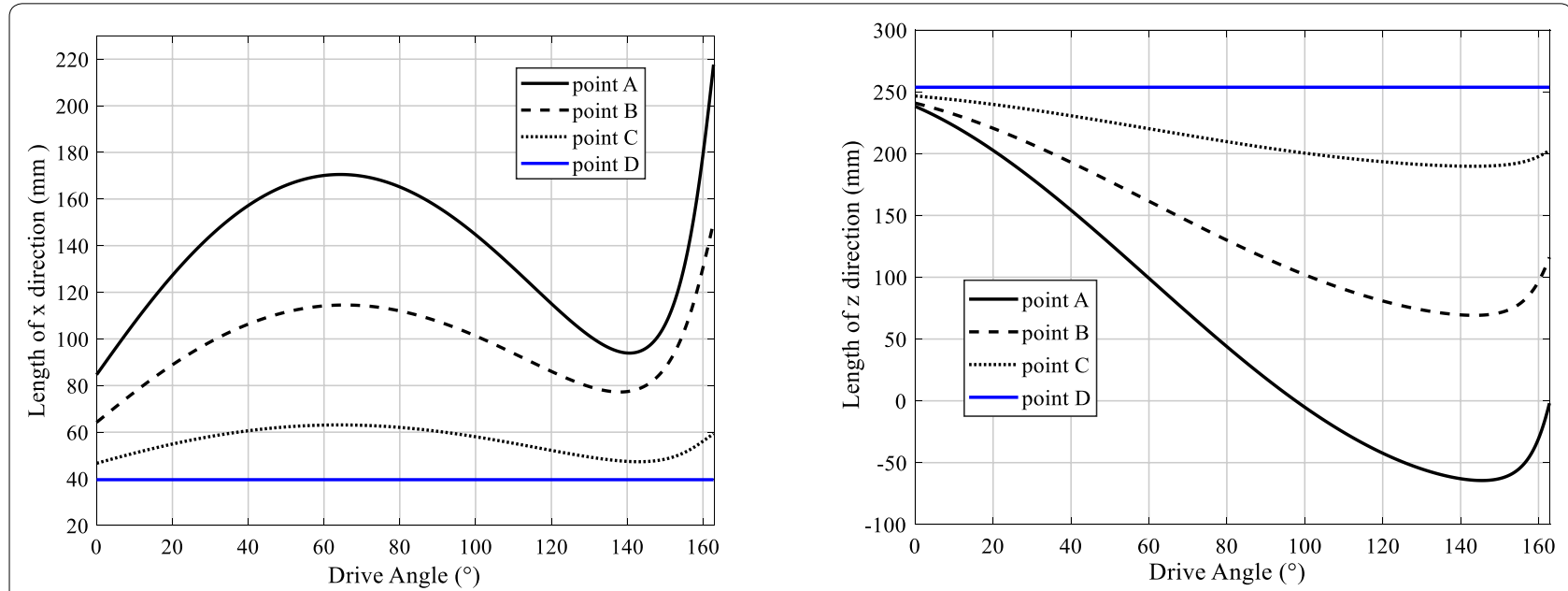

Figure 12. Motion trajectories of scissor points
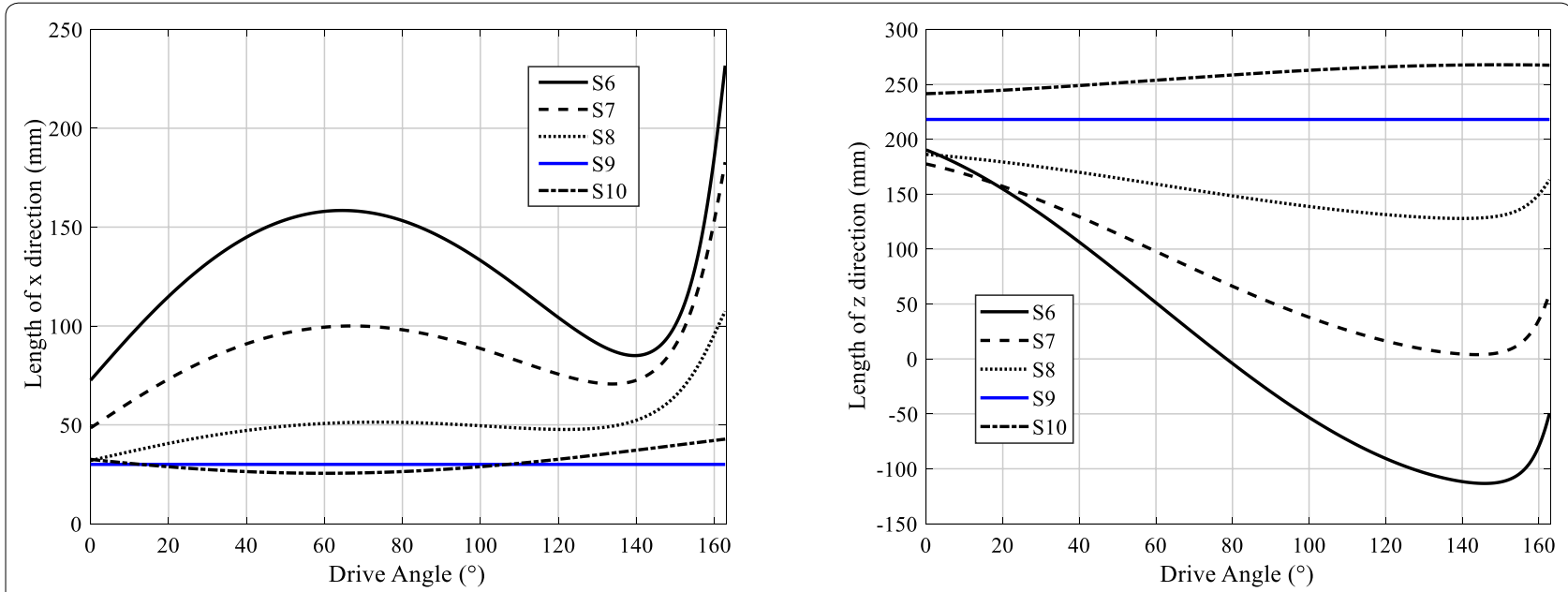

Figure 13. Motion trajectories of scissor points

Figures 11, 12, and 13 show the movement trajectories of the upper end-point, scissor point, and lower end-point of the planar scissor mechanism during the deployment, respectively. Because rod 5-9 is set to be fixed, end-points $S_{5}$ and $S_{9}$ and scissor point $D$ show no change in their positions, which appear as a straight line in the three figures. It can be seen that the motion path of the planar scissor mechanism is continuous and smooth from the folded state to the deployed state, and the deployment process is feasible and controllable.

The final deployed state of PSDA cannot ensure that all the lower ends of the planar scissor mechanism are coincident with the paraboloid, and will be uncertain deviation because of the errors in the rotation angle and machining. The calculated coordinate value in the deployed state is compared with the theoretical coordinate value. The following data is calculated separately: (1) The difference between theoretical and calculated values of the $x$ coordinate is represented as $\Delta u$. It

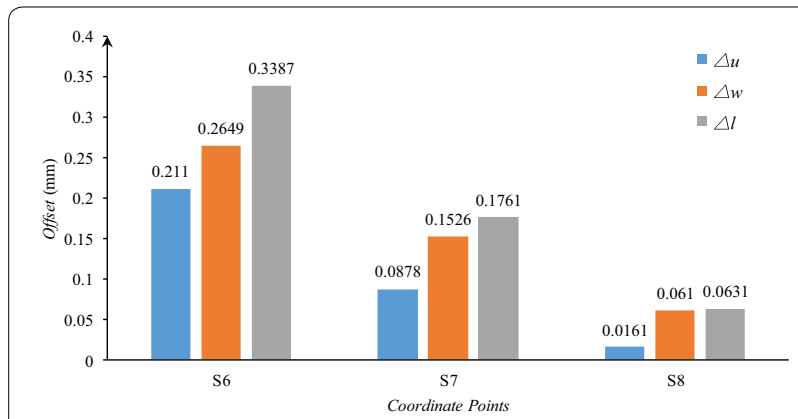

Figure 14. Offset between calculated coordinates and theoretical coordinates 
Table 2. Rod lengths of spatial triangular polar scissor unit (mm)

\begin{tabular}{lcccrr}
\hline Rod number & No. 1 & No. 2 & No. 3 & No. 4 & No. 6 5 \\
\hline Part 1 & 53.6269 & 54.663 & 55.4085 & 45.6324 & 52.5127 \\
Part 2 & 44.3646 & 54.6929 & 48.7305 & 43.6191 & 55.3153 \\
Total length & 97.9915 & 109.3559 & 104.139 & 89.2515 & 107.828 \\
\hline
\end{tabular}

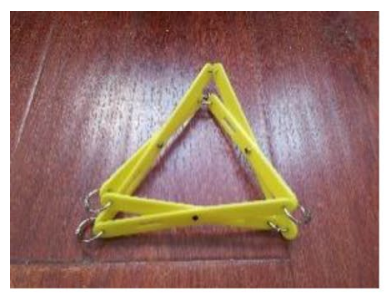

a

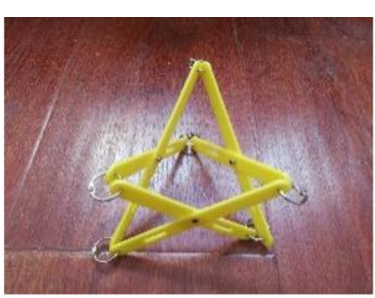

b

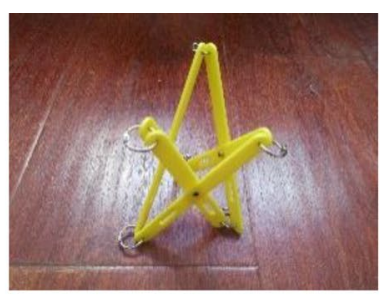

c

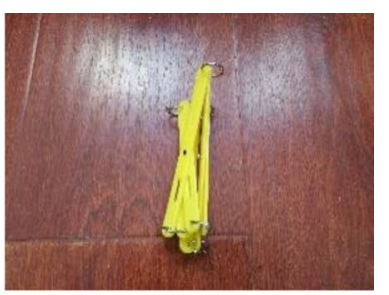

d

Figure 15. Motion sequence of spatial triangular polar scissor unit

is positive when the value of the former is greater than that of the latter. (2) The difference between theoretical and calculated values of the $y$ coordinate is represented as $\Delta w$. It is positive when the value of the former is greater than that of the latter. (3) The distance between theoretical coordinate and calculated coordinate values is represented as $\Delta l$. There is no motion error to rod 5-9 because of the fixed location during the movement. Therefore, the error relationship between calculated coordinates and theoretical coordinates can be measured based on rod 5-9. Express the ideal coordinate positions as $\left(x_{T}(i) y_{T}(i)\right)$, the calculated coordinate positions as $\left(x_{c}(i) y_{c}(i)\right)$, the difference formulas are as follows:

$$
\begin{aligned}
& \left\{\begin{array}{l}
\Delta u=x_{T}(i)-x_{C}(i), \\
\Delta w=y_{T}(i)-y_{C}(i), \\
\Delta l=\sqrt{\left(x_{T}(i)-x_{C}(i)\right)^{2}+\left(y_{T}(i)-y_{C}(i)\right)^{2}},
\end{array}\right. \\
& i=6,7,8 .
\end{aligned}
$$

The calculated coordinate values of $S_{6}, S_{7}, S_{8}$, and $S_{9}$ obtained by simulations are $(299.789,19.7351)$, (221.9122, 110.3274), (119.9839, 187.939) and $(30,218)$ respectively. Figure 14 shows the offset between calculated coordinates and theoretical coordinates for $S_{6}, S_{7}$, and $S_{8}$.

\section{Example and Prototype}

\subsection{Spatial Triangle Polar Scissor Unit}

The length of the spatial triangle scissor unit is designed to verify the correctness of (9) and (10). The set of rod lengths based on the Monte Carlo method is presented in Table 2. The portion form the upper-end of the scissor

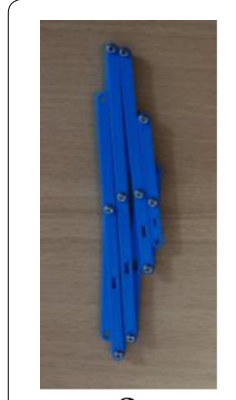

a

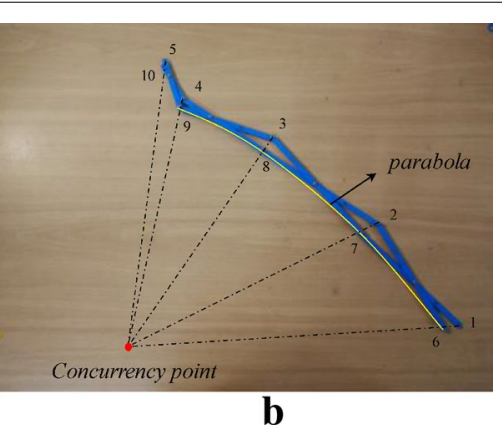

b
Figure 16. Planar polar scissor mechanism: a folded state, $\mathbf{b}$ deployment state

Table 3. Rod sizes of top and bottom rings ( $\mathrm{mm}$ )

\begin{tabular}{lll}
\hline Rod sizes & Top ring & Bottom ring \\
\hline Part 1 & 16.22 & 47.34 \\
Part 2 & 15.98 & 60.47 \\
Total length & 32.2 & 107.82 \\
Number & 18 & 36 \\
\hline
\end{tabular}

bar to the scissor point is called Part 1, and the portion from the lower-end point to the scissor point is called Part 2. The spatial polar scissor unit realizes the space movement, and connections with circular rings are used between the scissor units instead of spherical hinges to reduce the processing difficulty. Figure 15 shows the motion sequence of deployment for a spatial triangular scissor unit. The experimental result shows that the spatial triangle scissor unit can achieve complete 


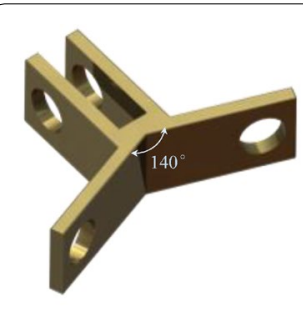

a Hinge for top ring

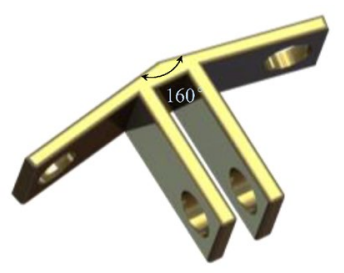

b Hinge for bottom ring

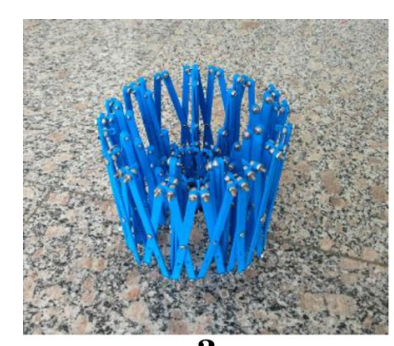

a

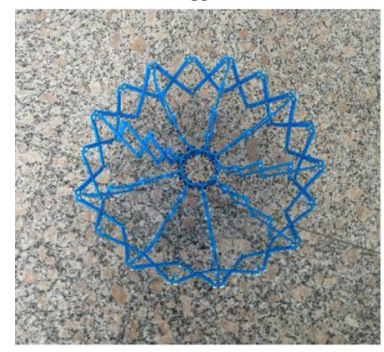

c

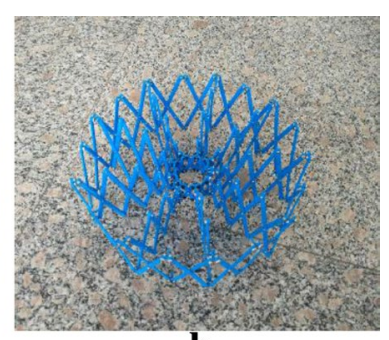

b

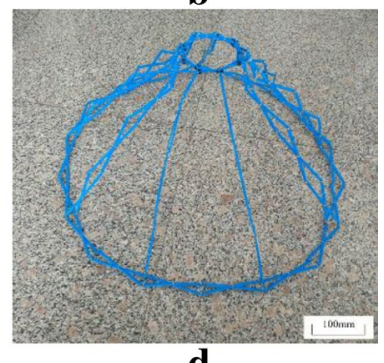

d
Figure 18. Motion sequence of deployment for PSDA

deployment, and the deployment process can be smooth, which verifies the correctness of the RM.

\subsection{Planar Polar Scissor Curve}

The planar scissor mechanism has been designed on the basis of motion simulation and error analysis in Section 3.2. The data on rod lengths are presented in Table 1. Figure 16 shows the folded and deployed states of the planar scissor mechanism. The deployment process is consistent with the simulation analysis in Section 3.2.

\subsection{Polar Scissor Deployable Antenna}

PSDA is a spatial closed-loop structure consisting of a top ring, a bottom ring and planar scissor mechanisms. Because the diameter of the bottom ring is considerably larger than that of the top ring, the rod lengths of the bottom ring are bound to be significantly larger than that of the top ring. To reduce the difference between the lengths of the top and bottom rings and the design of the overall structure, the bottom ring is designed as a regular octadecagon polar scissor unit, and the top ring is designed as a nonagon polar scissor unit. Therefore, nine sets of planar scissor mechanisms are machined to connect the top and bottom rings. The dimensions of the top and bottom rings are designed using the method described in Section 3.1.1. The design results are presented in Table 3. The method that increases the number of planar scissor mechanisms can be chosen to achieve better surface fitting. Accordingly, the number of polygon sides for top and bottom rings will increase.

In the PSDA model, the connecting parts between the rods are represented by dots. These dots need to be converted into objects that can be fabricated with shapes and thicknesses. At the end of the scissor device, the joint connects a plurality of scissor devices in a threedimensional configuration, and each scissor device typically rotates about a different axis during deployment. Fabricating such a structure requires high-tech ball joints that considerably increase the cost of the structure. On the contrary, based on the movement characteristics of PSDA, the hinge structures connecting the planar scissor mechanism and top/bottom ring will significantly reduce the manufacturing cost as shown in Figure 17. The assembly of all the parts ultimately constitutes the PSDA. Figure 18 shows the motion sequence of the deployment process for PSDA. Experiments show that the final state of deployment can achieve the desired effect.

\section{Conclusions}

In this study, spatial and planar polar scissor units were combined on the basis of relevant properties of the polar scissor unit. A novel method for designing the aspherical geometry of a space reflector antenna by the polar scissor unit was proposed. The main conclusions are as presented below:

1. The concurrency and deployability equations for spatial polar scissor units were derived by two methods: the RM and VM. The constraint equations of the spatial $n$-sided polar scissor unit were summarized, and their validities were experimentally.

2. A new design method of PDA that combined planar and spatial polar scissor units was proposed, and a new mechanism, called PSDA was designed on the basis of the above equations. The method of releasing the curve constraint that locates at the end-point of the plane scissor mechanism solved the problem that there may be no reasonable rod length.

3. The rod lengths of the planar scissor mechanism were designed, and the kinematic simulation and error analysis were carried out. The analysis results showed that the deployed PSDA can achieve a better fit with a rotating paraboloid. 
4. A prototype of PSDA was fabricated, and a deployment experiment was realized to verify the feasibility of the design method.

It is worth noting that the selection of concurrency point $P$ was arbitrary in this paper. $P$ was selected at the origin of the coordinate system for ease of calculation. In fact, the selection of $P$ plays a crucial role in the design of rod lengths. The rod length can be optimized by $P$. We will focus on the optimization of rod lengths for this new spatial surface design method in our next study. Furthermore, by considering the operating characteristics of the space reflector antenna, improving the profile accuracy of this antenna will be one of the research objectives in the future.

\section{Acknowledgements}

The authors sincerely thanks to Professor Yangmin Li of The Hong Kong Polytechnic University for his critical discussion and reading during manuscript preparation.

\section{Authors' contributions}

PZ was in charge of the whole trial; PZ wrote the manuscript; $\mathrm{LL}, \mathrm{CW}$ and KC assisted with sampling and laboratory analyses; $Y L$ modified the manuscript. All authors read and approved the final manuscript.

\section{Authors' Information}

Pengyuan Zhao, born in 1994, is currently a PhD candidate at State Key Laboratory of Robotics, Shenyang Institute of Automation, Chinese Academy of Science, China.

Jinguo Liu, born in 1978, is currently a professor and a PhD candidate supervisor at Shenyang Institute of Automation, Chinese Academy of Science, China.

Chenchen Wu, born in 1990, is currently an associate professor at Shenyang Institute of Automation, Chinese Academy of Science, China.

Yangmin Li, is currently a professor and a PhD candidate supervisor at The Hong Kong Polytechnic University, China.

Keli Chen, born in 1991, is currently an engineer at Shenyang Institute of Automation, Chinese Academy of Science, China.

\section{Funding}

Supported by National Key R\&D Program of China (Grant No. 2018YFB1304600), National Natural Science Foundation of China (Grant No. 51775541), CAS Interdisciplinary Innovation Team of China (Grant No. JCTD2018-11), Hundred-Talent Program (Chinese Academy of Sciences) (Grant No. Y8A3210304)

\section{Competing interests}

The authors declare no competing financial interests.

\section{Author Details}

${ }^{1}$ State Key Laboratory of Robotics, Shenyang Institute of Automation, Chinese Academy of Science, Shenyang 110016, China. ${ }^{2}$ Institutes for Robotics and Intelligent Manufacturing, Chinese Academy of Sciences, Shenyang 110169, China. ${ }^{3}$ University of Chinese Academy of Sciences, Beijing 100049, China. ${ }^{4}$ Department of Industrial and Systems Engineering, The Hong Kong Polytechnic University, Hong Kong 999077, China.

Received: 31 January 2020 Revised: 30 August 2020 Accepted: 6 October 2020

Published online: 20 October 2020

\section{References}

[1] C Shi, H Guo, M Li, et al. Conceptual configuration synthesis of linefoldable type quadrangular prismatic deployable unit based on graph theory. Mechanism and Machine Theory, 2018, 121: 563-582.

[2] X Song, Z Deng, H Guo, et al. Networking of Bennett linkages and its application on deployable parabolic cylindrical antenna. Mechanism and Machine Theory, 2017, 109: 95-125.

[3] S Yang, Y Li. Kinematic analysis of deployable parallel mechanisms. Proceedings of the Institution of Mechanical Engineers, Part C: Journal of Mechanical Engineering Science, 2019: 095440621882532.

[4] S Yuan, B Yang. The fixed nodal position method for form finding of highprecision lightweight truss structures. International Journal of Solids and Structures, 2019, 161: 82-95.

[5] TTakano, K Miura, M Natori, et al. Deployable antenna with 10-m maximum diameter for space use. IEEE Transactions on Antennas and Propagation, 2004, 52(1): 2-11.

[6] Y Gu, J Du, D Yang, et al. Form-finding design of electrostatically controlled deployable membrane antenna based on an extended force density method. Acta Astronautica, 2018, 152: 757-767.

[7] R Nie, B He, D H Hodges, et al. Integrated form finding method for mesh reflector antennas considering the flexible truss and hinges. Aerospace Science and Technology, 2019, 84: 926-937.

[8] R Liu, H Guo, R Liu, et al. Shape accuracy optimization for cable-rib tension deployable antenna structure with tensioned cables. Acta Astronautica, 2017, 140: 66-77.

[9] T Langbecker. Kinematic analysis of deployable scissor structures. International Journal of Space Structures, 1999, 14(1): 1-15.

[10] E P Piñero. Project for a mobile theatre. Architectural Design, 1961, 12(1): 154-155.

[11] F Escrig, J P Valcarcel. Great size umbrellas with expendable bar structures. Proceedings of the 1st International Conference on Lightweight Structures in Architecture, 1986: 676-681.

[12] F Maden, K Korkmaz, Y Akgün. A review of planar scissor structural mechanisms: geometric principles and design methods. Architectural Science Review, 2011, 54(3): 246-257.

[13] F Escrig. Expandable space frame structures. Third International Conference on Space Structures, Guildford, UK, 1984: 845-850.

[14] R Liu, H Guo, R Liu, et al. Structural design and optimization of large cable-rib tension deployable antenna structure with dynamic constraint. Acta Astronautica, 2018, 151: 160-172.

[15] Y Chikahiro, I Ario, P Pawlowski, et al. Optimization of reinforcement layout of scissor-type bridge using differential evolution algorithm. Computer-Aided Civil and Infrastructure Engineering, 2019, 34(6): 523-538.

[16] E Medzmariashvili, Sh Tserodze, N Tsignadze, et al. A new design variant of the large deployable space reflector. Earth and Space 2006-10th Biennial International Conference on Engineering, Construction, and Operations in Challenging Environments, Houston, United States, March 5-8, 2006: 6.

[17] W Bettini, J Quirant, J Averseng, et al. Self-deployable geometries for space applications. Journal of Aerospace Engineering, 2019, 32(1): 1-12.

[18] C Q Gao, H L Huang, B Li, et al. Design of a truss-shaped deployable grasping mechanism using mobility bifurcation. Mechanism and Machine Theory, 2019, 139: 346-358.

[19] L Alegria Mira, R Filomeno Coelho, A P Thrall, et al. Parametric evaluation of deployable scissor arches. Engineering Structures, 2015, 99: 479-491.

[20] L I W Arnouts, T J Massart, N De Temmerman, et al. Computational modelling of the transformation of bistable scissor structures with geometrical imperfections. Engineering Structures, 2018, 177: 409-420.

[21] B Li, S M Wang, C J Zhi, et al. Analytical and numerical study of the buckling of planar linear array deployable structures based on scissor-like element under its own weight. Mechanical Systems and Signal Processing, 2017, 83: 474-488.

[22] A Koumar, TTysmans, N D Merman. Structural design of barrel vault shaped scissor structures for disaster relief. Journal of the International Association for Shell and Spatial Structures, 2018, 59(3): 171-182.

[23] Y Akgün, C J Gantes, K E Kalochairetis, et al. A novel concept of convertible roofs with high transformability consisting of planar scissor-hinge structures. Engineering Structures, 2010, 32(9): 2873-2883.

[24] S Lu, D Zlatanov, X Ding, et al. A new family of deployable mechanisms based on the Hoekens linkage. Mechanism and Machine Theory, 2014, 73: 130-153. 
[25] A S K Kwan. Parabolic pantographic deployable antenna (PDA). International Journal of Space Structures, 1995, 10(4): 195-203.

[26] Z You. Deployable structure of curved profile for space antennas. Journal of Aerospace Engineering, 2000, 13(4): 139-143.

[27] Z You, S Pellegrino. Cable-stiffened pantographic deployable structures. II-Mesh reflector. AlAA Journal, 1997, 35(8): 1348-1355.

[28] K Roovers, N De Temmerman. Deployable scissor grids consisting of translational units. International Journal of Solids and Structures, 2017, 121: 45-61.
[29] K Roovers, N De Temmerman. Geometric design of deployable scissor grids consisting of generalized polar units. Journal of the International Association for Shell and Spatial Structures, 2017, 58(3): 227-238.

[30] Y Chen, L Fan, J Feng. Kinematic of symmetric deployable scissor-hinge structures with integral mechanism mode. Computers \& Structures, 2017 191: 140-152.

[31] C Hoberman. Reversibly expandable doubly-curved truss structure: US, 4942700, 1990-07-24.

\section{Submit your manuscript to a SpringerOpen ${ }^{\circ}$ journal and benefit from:}

- Convenient online submission

- Rigorous peer review

- Open access: articles freely available online

- High visibility within the field

- Retaining the copyright to your article 